\title{
Perirhinal cortex and visual discrimination learning in the rat
}

\author{
PENNY E. MACHIN and M. J. EACOTT \\ Science Laboratories, Durham, England
}

\begin{abstract}
Sixteen Dark Agouti rats learned two sets of concurrent visual discriminations in a computeroperated apparatus at different time intervals (approximately 8 and 3 weeks) prior to perirhinal ablation or control surgery. Following surgery, the perirhinal animals demonstrated a deficit in reacquisition of the discriminations learned preoperatively. There was no evidence that this deficit was temporally graded, but it was limited to initial postoperative performance. Analysis of the speed to approach the stimulus or to retrieve the reward revealed no differences between the groups, suggesting that non-mnemonic factors were not playing a significant role in the deficit. In order to test postoperative acquisition of visual associative information, all animals learned a new and larger set of concurrent visual discriminations, thereby increasing demands on the processes of object identification. The perirhinal lesioned animals were not impaired in acquiring the new set of discriminations. These results suggest that the deficit may be transient.
\end{abstract}

Both electrophysiological and behavioral studies suggest that mechanisms within the rhinal cortex contribute to visual recognition memory (Fahy, Riches, \& Brown, 1993; D. Gaffan \& Murray, 1992; Meunier, Bachevalier, Mishkin, \& Murray, 1993; Miller, Li, \& Desimone, 1993). Behavioral studies have demonstrated distinct impairments in visual recognition memory tasks, such as the delayed match- or non-match-to-sample task, following rhinal ablation in both the monkey (D. Gaffan \& Murray, 1992) and the rat (Mumby \& Pinel, 1994). In the monkey ablation of the perirhinal cortex alone is sufficient to cause this deficit and has therefore been identified as an important component in visual recognition memory performance (Meunier et al., 1993).

However, the behavioral effects of perirhinal ablation on other visual tasks are less certain. There are contradictory reports of both impaired (Kornecook, Lui, Duva, Anzarat, \& Pinel, 1995) and unimpaired (Astur, Mumby, $\&$ Sutherland, 1995) retention of visual discrimination learning following perirhinal ablation in the rat. A more recent study designed to clarify this issue showed that perirhinal ablation produced a deficit in retention of concurrent visual discriminations acquired prior to surgery (Eacott, 1998). The same animals then went on to learn a new set of discriminations at a rate comparable to that of control animals, suggesting that a dissociation exists between anterograde and retrograde visual associative memory processes following perirhinal cortex ablation in the rat. However, Buckley and Gaffan (1997) have suggested

We gratefully acknowledge the help of S. Nagle, who provided technical assistance during surgery, and Shirley Whiteley, who processed the histology. P.E.M. is in receipt of a studentship from the Department of Psychology, Durham University. Correspondence should be addressed to M. J. Eacott, Department of Psychology, Science Laboratories, South Road, Durham DH1 3LE, England (e-mail: m.j.eacott@durham.ac.uk). that in monkeys, perirhinal cortex ablation impairs both the retention of old discriminations and the ability to acquire new visual discrimination learning. In their study, the impairment in new learning was revealed by increasing the number of stimuli in the discrimination task during acquisition (Buckley \& Gaffan, 1997). This finding is reminiscent of the report that performance of delayed match to sample in the monkey is impaired by rhinal cortex ablation only when the size of the stimuli set used is relatively large (Eacott, Gaffan, \& Murray, 1994). This pattern of impairments has led to the suggestion that loss of accurate object identification disrupts performance of both recognition and associative memory tasks following perirhinal ablation in the monkey (Buckley \& Gaffan, 1997; Eacott \& Heywood, 1995). Although it is possible that perirhinal ablation affects new learning in monkeys and rats differently, it is also possible that the rat's postoperative learning capacity has not been sufficiently taxed to reveal a similar deficit following perirhinal lesions. Thus, the existing rat literature is not extensive enough to reveal the exact nature of impairments in visual associative memory tasks following perirhinal ablation. In particular, it is unclear whether perirhinal ablation produces a deficit in retention of visual associative information acquired prior to surgery, while leaving new learning intact.

It has also been suggested that the perirhinal cortex may contribute to the consolidation of associative memories by virtue of its extensive cortical and subcortical connections (Squire \& Zola-Morgan, 1991). For example, perirhinal back projections to area TE appear to be necessary for successful visual paired associate learning (Higushi \& Miyashita, 1996). If this is the case, it may be expected that the retrograde memory impairments following perirhinal ablation are temporally graded, as in human amnesic patients (Warrington \& McCarthy, 1988). However, behavioral evidence to ascertain the role of the perirhinal cortex 
in consolidation processes has so far proved inconclusive. There are reports of combined lesions to the hippocampus and rhinal cortex in the monkey resulting in a temporally graded retrograde amnesia for object discriminations, with better retention of items learned 16 weeks prior to surgery, relative to items learned 1 week prior (Zola-Morgan \& Squire, 1990). Subsequent studies with rats in spatial memory (Weisand, Astur, \& Sutherland, 1996), object place memory (Koerner, Thomas, Weisand, \& Sutherland, 1996), and object discrimination tasks (Astur, Mumby, Weisand, \& Sutherland, 1994) all failed to replicate these findings with lesions restricted to the hippocampus, suggesting that the deficit is dependent on the rhinal cortex ablation. However, studies focusing on the rhinal cortex itself have produced contradictory results. There is evidence that perirhinal ablation, fornix transection, or both of these combined in the rat results in a temporally graded retrograde amnesia, with better retention of visual discriminations learned 6-8 weeks prior to surgery than discriminations learned 1-3 weeks prior (Wiig, Cooper, \& Bear, 1996). However, similar studies in rats (Astur et al., 1995) and in primates (Thornton, Rothblat, \& Murray, 1997) have failed to show a temporally graded retrograde effect following perirhinal ablation. Thus, the retrograde effects of perirhinal cortex lesions are unclear.

The aim of the present experiments was therefore to further investigate the effects of perirhinal cortex ablation on pre- and postoperatively learned visual discrimination tasks in the rat. Since it has been suggested that perirhinal ablation disrupts object identification processes (Buckley \& Gaffan, 1997; Eacott \& Heywood, 1995), a difficult version of the concurrent visual discrimination task was used in which any rewarded stimulus $(\mathrm{S}+)$ can be presented with any unrewarded stimulus $(\mathrm{S}-)$ rather than stimuli always being presented in fixed pairings. With constant pairings, one salient feature of the $\mathrm{S}+$ or $\mathrm{S}-$ may be sufficient to discriminate between the two stimuli. Thus, an animal may adopt a strategy of using a single feature of stimuli pairs. However, with a variety of $S+/ S-$ pairings, the animal has to distinguish the $S+$ from a number of $\mathrm{S}-\mathrm{s}$ with which it may be paired. A single feature that is sufficient to distinguish between the $\mathrm{S}+$ and a particular S - may be insufficient when there are multiple $\mathbf{S}-\mathbf{s}$. This version of the task therefore assumes more demands on visual identification processes than a twochoice discrimination with constant stimuli pairings, and thus may be more likely to reveal a postoperative deficit.

In Experiment 1, we asked whether perirhinal cortex ablation in the rat produces a retrograde amnesia for visual associative information and whether this deficit is temporally graded. The animals learned two sets of concurrent visual discriminations at different time intervals (approximately 8 and 3 weeks) prior to either a bilateral perirhinal lesion or sham surgery. The order of testing postoperative reacquisition of the two sets of discriminations was balanced to control for any transience in postoperative deficits. The testing took place in a computercontrolled Y-maze to minimize testing variances associ- ated with traditional testing apparati. In Experiment 2, we examined the effect of perirhinal ablation on postoperative acquisition of new visual associative information. In Experiment 2, the animals that had successfully completed Experiment 1 learned a new set of concurrent visual discriminations in which the number of stimuli was gradually increased to tax the proposed anterograde memory processes of the perirhinal cortex of the rat.

\section{EXPERIMENT 1}

\section{Method}

Subjects. Twenty male Dark Agouti rats (Bantin \& Kingman, Hull, U.K.) were used in this experiment. They were housed individually in diurnal conditions (12:12-h light:dark cycle) and were tested during the light period of the cycle. Except when in the testing apparatus, the rats had free access to water and were food deprived prior to testing to approximately $80 \%$ of normal body weight. They were tested 5 days a week. Two rats failed to reach criterion on the tests used and were removed from the study, and another 2 died of respiratory infection in the days following surgery. Overall, 16 rats completed Experiment 1, 7 had bilateral sham surgery, and 9 had bilateral perirhinal lesions.

Apparatus. The apparatus used was a computer-operated $Y$ maze. Since this maze is described fully in E. A. Gaffan and Eacott (1995), it will be described only briefly here. Each of the three arms of the maze holds two display screens, separated by a food well. In any given trial, two different stimuli appear in different arms of the maze, approach to only one of which will elicit a food reward. Infrared beams crossing the arms of the maze will detect an approach to the monitors displaying the rewarding stimulus. If a correct approach is detected, the food well is illuminated and a pellet dispensed. When the pellet is retrieved, the stimuli presentation ends and a new trial begins. If the animal approaches the nonrewarded stimulus, it is detected by the infrared beams; the stimuli presentation then ends and a new trial begins.

Original composition of the three different stimuli sets used was by pseudorandom selection from a large number of computergenerated abstract scenes resembling junk objects. The only restriction was that a stimulus could not appear in more than one set. All three stimulus sets had been used in a previous study (Eacott, 1998). A full description of the stimuli and their generation can be found in E. A. Gaffan and Eacott (1995).

Preoperative training. Initial training involved approaching simple stimuli presented in the maze for food rewards, as described by E. A. Gaffan and Eacott (1995). When this was accomplished, training in the concurrent visual discrimination task began. The rats learned two sets of stimuli, each comprising five pairs of stimuli. Within each pair there was a rewarded stimulus $(\mathrm{S}+)$ and an unrewarded foil $(\mathrm{S}-$ ). First, the animals were presented with just one pair of stimuli. When they reached a criterion of $80 \%$ correct within a single session, another pair of stimuli was introduced. Subsequent pairs were introduced in the same way until the animals were discriminating between five pairs of stimuli to a criterion of $75 \%$ correct. At all stages of training, stimuli presentations were not confined to the initial pairings, so that a given $\mathrm{S}+$ could be presented with any one of the currently used $S-S$.

A 2-week rest period was introduced between reaching criterion on Set 1 and beginning training on Set 2. Therefore, Set 1 was learned approximately 8 weeks prior to surgery, and Set 2 at approximately 3 weeks prior to surgery. Animals were then pseudorandomly assigned to experimental or control groups and given a 3week rest period before surgery.

Surgery. Surgery was performed on all animals, either bilateral perirhinal lesion by aspiration or a sham control surgery. Each rat 
was anesthetized using halothane; its head was shaved and the rat was positioned in a stereotaxic head holder that allowed rotation of the head. An incision was made in the skin, the temporal muscle retracted, and an area of the skull was removed over the rhinal sulcus, approximately 4-7 $\mathrm{mm}$ posterior to bregma. In the perirhinal group of animals, the dura was cut to allow access to the underlying cortex and a lesion was made by aspiration with the aid of an operating microscope. For both perirhinal and sham animals, the temporal muscle was returned to place and the procedure was performed contralaterally. The scalp was then closed using wound clips, and antibacterial wound powder was applied. Each animal received $5 \mathrm{ml}$ of saline, $0.3 \mathrm{ml}$ of respiratory stimulant (milolphyline), and $0.5 \mathrm{ml}$ of analgesic (vetergesic) subcutaneously. Postsurgical recovery was normal for all but 2 animals that developed fatal respiratory infections.

For histological purposes, at the end of testing the rats were perfused intracardially with a $5 \%$ formal saline solution; their brains were removed, embedded in wax, and coronally sectioned into 10 $\mu \mathrm{m}$ slices. Every 10th section was stained with cresyl violet (Nissl stain).

Postoperative testing. All animals had a 2-week rest period after surgery prior to postoperative testing. They were then pseudorandomly assigned to two groups for testing postoperative retention of Sets 1 and 2. Animals in Sequence $A$ were tested for reacquisition of Set 1, followed by Set 2, whereas animals in Sequence B were tested in the reverse order. Performance in postoperative retention was assessed by calculating the number of trials and errors accumulated in reattaining a criterion of $75 \%$ correct choices made in a single session of up to 100 trials. Reacquisition rates were used to assess retention, since a major determinant of the rate of reacquisition is the degree of retention of information acquired prior to surgery, along with the ability to perform non-mnemonic demands of the task and relearn what has been forgotten. During both preoperative acquisition and postoperative reacquisition, two other measures were recorded in order to measure possible non-mnemonic effects of surgery. First, the stimulus response time was measured as the time between stimulus onset and the rat entering the arm (breaking the beam) on correct trials. Second, the reward response time was measured as the time between reward delivery and reward collection (opening the hinged door of the food magazine).

\section{Results}

Histological results. Histological analysis revealed that in all cases the perirhinal lesions were essentially as intended, extending approximately $4-7 \mathrm{~mm}$ posterior to Bregma. Figure 1 shows a representative section from Animal R5. The perirhinal cortex was clearly lesioned in all cases; in some animals there was a small degree of intact tissue toward the extreme dorsal extent of the intended lesion. There was no evidence of damage to the neighboring entorhinal cortex, although in some cases there was slight intrusion into adjacent area TE of inferotemporal cortex. In all cases, there was some intact tissue toward the extreme caudal extent of the lesion, thereby excluding the possibility of unintended damage extending into postrhinal cortex. Figure 2 shows a representative section from a relatively large lesion (R8) and a small lesion (R45).

Preoperative learning. The two groups formed, the perirhinal and sham groups, did not differ significantly in the number of trials [Set $1, t(14)=0.37, p=.711$; Set 2 , $t(14)=1.02, p=.324]$ or errors [Set $1, t(14)=0.22, p=$
.826 ; Set $2, t(14)=0.54, p=.598]$ made in attaining criterion preoperatively. Both groups found Set 1 significantly more difficult to learn than Set 2 [trials, $t(15)=$ $12.63, p \leq .001$; errors, $t(15)=10.89, p \leq .001]$. Although this disparity was also found in an earlier study (Eacott, 1998), it was unclear then whether Set 1 was actually more difficult to acquire or whether differences in acquisition were due to the animals' previous experience in another visual learning task. The present study suggests that there is a real difference in difficulty between the sets. We also recorded the response time to the onset of stimuli presentation (from stimulus onset to the rat entering the correct arm) and the collection of the reward (from reward delivery to reward collection). Preoperatively, there were no significant differences between the groups for either set on response time to stimulus onset [Set $1, F(1,15)=$ $1.142, p=.303$; Set $2, F(1,15)=0.003, p=.956]$ or to reward delivery [Set $1, F(1,15)=3.148, p=.098$; Set 2, $F(1,15)=0.352, p=.562]$.

Postoperative reacquisition. The groups were pseudorandomly subdivided for postoperative testing: Those in Sequence A were tested for retention in the order in which the sets were learned, whereas animals in Sequence B were tested in the reverse order. Postoperative retention was assessed by comparing the number of trials and errors accumulated in reacquisition of the two sets of discriminations to criterion.

The effect of group, set, and sequence on the number of trials taken to reattain criterion for both sets of discriminations is shown in Figure 3. The number of trials and errors to criterion were analyzed by $2 \times 2 \times 2$ (group $X$ set $\times$ sequence) analyses of variance (ANOVAs) with one repeated measure. Overall there was no significant difference between reacquisition of Set 1, learned approximately 8 weeks prior to surgery, and Set 2 , learned approximately 3 weeks prior [set: trials, $F(1,12)=0.01$, $p=.931$; errors, $F(1,12)=0.14, p=.72]$. There was also no significant interaction between group and set [trials, $F(1,12)=0.01, p=.39$; errors, $F(1,12)=2.13, p=.17]$. The mean number of trials to criterion was 179 for the perirhinal group and 180 for the control group; therefore there was no significant difference between the two groups in terms of overall performance [group: trials, $F(1,12)=$ $0.01, p=.93$; errors, $F(1,12)=0.15, p=.70]$. The main effect of sequence was also not statistically significant [sequence: trials, $F(1,12)=3.22, p=.10$; errors, $F(1,12)=$ $1.31, p=.27]$, nor was the interaction between set and sequence [trials, $F(1,12)=0.23, p=.23$; errors, $F(1,12)=$ $3.09, p=.10]$. However, the interaction between group and sequence was significant [trials, $F(1,12)=11.73, p=$ .005 ; errors, $F(1,12)=6.84, p=.02]$. As Figure 3 illustrates, the sequence of testing affected the two groups in opposing ways: The perirhinal animals demonstrated a marked improvement in performance in the second set in which they were tested relative to the first [trials, $t(8)=$ $3.11, p=.01$; errors, $t(8)=2.24, p=.05$ ], whereas the per- 

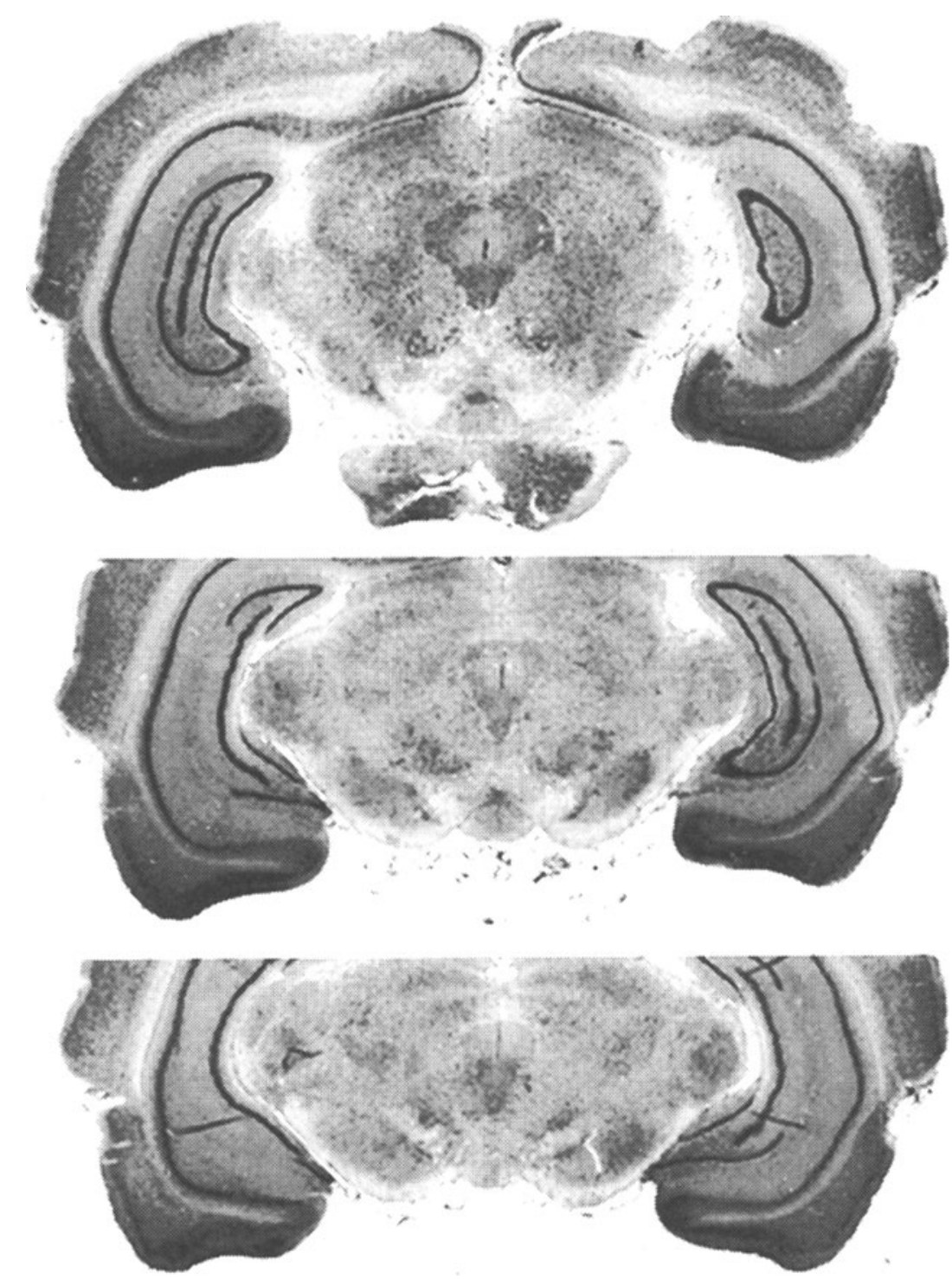

Figure 1. A representative section of a bilateral perirhinal lesion from Animal R5.

formance of the sham animals did not improve [errors, $t(6)=1.52, p=.177$ ] and may have even declined [trials, $t(6)=2.435, p=.05]$.

To investigate this effect further, a percent savings score was calculated for each rat in order to measure postoperative reacquisition of the first set tested postoperatively relative to reacquisition of the second set tested. It was calculated on the basis of $(\mathrm{T} 1-\mathrm{T} 2) /(\mathrm{T} 1+\mathrm{T} 2) \times 100$, where $\mathrm{T} 1$ and $\mathrm{T} 2$ represent the number of trials (or errors) accumulated in reattaining criterion of the first set tested and the second set tested, respectively. The percentage saving score reflects the magnitude of the difference between the first and second set tested, ranging from $100 \%$ to $-100 \%$. A positive saving score reveals that the number of trials accumulated in reattaining criterion in the first set tested is higher than for the second. A negative saving score reveals the converse, a greater number of errors in the second set tested, relative to the first.

The savings score analysis revealed that the perirhinal animals pattern of reacquisition was significantly different from that of the control animals (Figure 4). A $2 \times 2$ (group $\times$ sequence) ANOVA on the percent savings of both the trials and errors to criterion for the first set tested relative to the second revealed a significant difference between the perirhinal and control groups [trials, $F(1,2)=$ $16.297, p=.002$; errors, $F(1,2)=8.941, p=.013]$. In contrast, there was no effect of sequence [trials, $F(1,2)=$ $0.377, p=.55$; errors, $F(1,2)=0.684, p=.424]$, nor any interaction between sequence and group [trials, $F(1,2)=$ $0.17, p=.688$; errors, $F(1,2)=0.788, p=.392]$. Further 


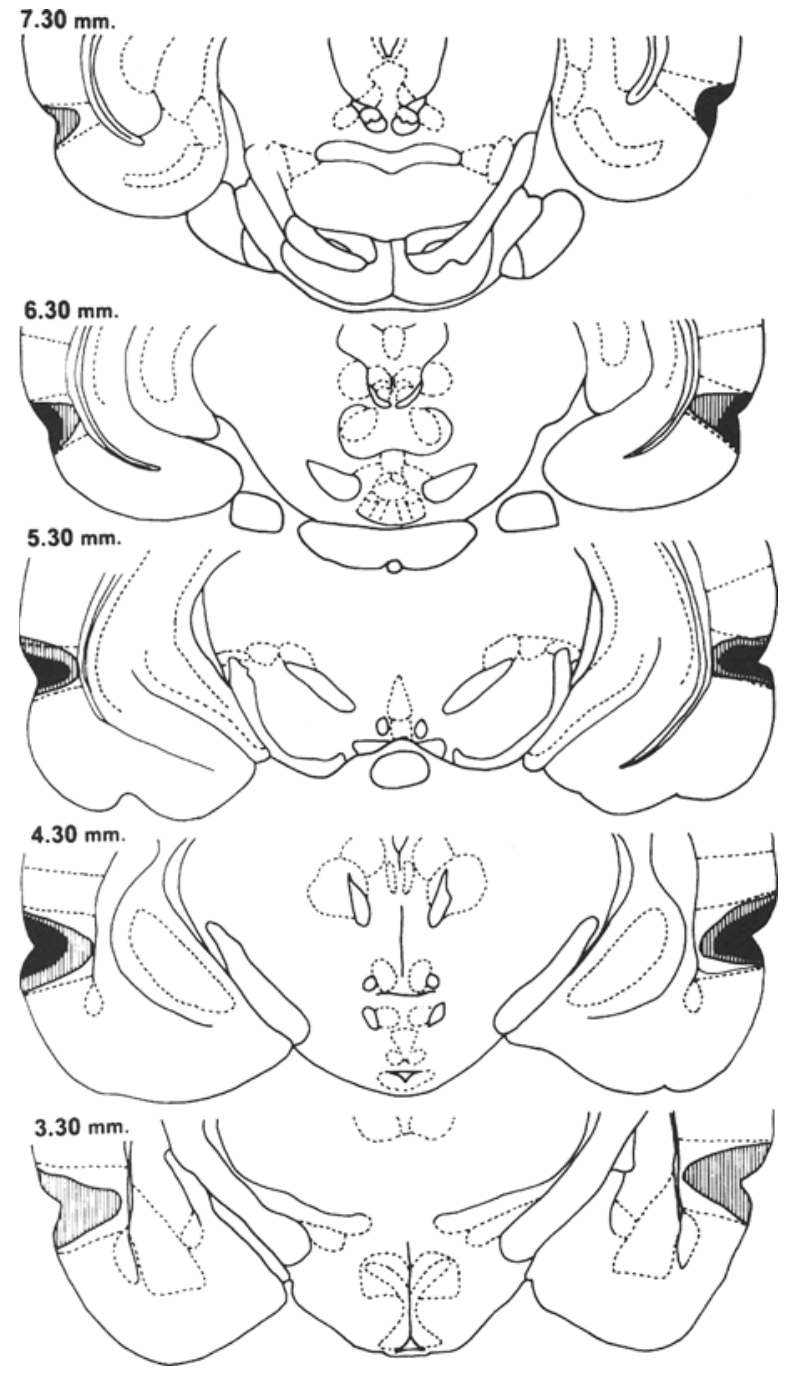

Figure 2. The extent of a relatively small (R8, black) and a relatively large ( $R 45$, vertical stripes) lesion drawn onto standard sections taken from Paxinos and Watson (1986).

analysis revealed that, overall, the savings scores of the perirhinal animals were significantly greater than zero [trials, $t(8)=4.104, p=.003$; errors, $t(8)=3.18, p=.01$ ], while the saving scores of the sham operated animals were not greater, and may have been significantly smaller, than zero [trials, $t(6)=2.455, p=.049$; errors, $t(6)=1.28, p=$ $.25]$. These results suggest that the perirhinal animals were significantly worse at the first tested set than the second, while for the sham operated animals the converse may have been true.

This analysis suggests that the perirhinal group may have had a deficit that was confined to the first tested set. Thus we reconsidered our previous analysis of retrograde effects, which was based on both postoperative sets, and which thus included data from sets in which the perirhinal animals were showing no impairment. In order to consider whether there was a differential effect of time of acquisition using data from only the postoperative period in which the perirhinal animals showed an impairment, a between-subjects comparison of only the first set tested was conducted. Thus we compared the reacquisition of Set 1 from Sequence A and Set 2 from Sequence B for both perirhinal and sham animals in a $2 \times 2$ (group $X$ set) ANOVA, thereby comparing immediate postoperative performances only in a between-animals comparison. As before, however, there was no effect of set [trials, $F(2,1)=0.399, p=.54$; errors, $F(2,1)=1.258, p=$ .284] nor any interaction between set and group [trials, $F(1,3)=1.152, p=.46$; errors, $F(1,3)=1.061, p=.323$ ] In this analysis, the effect of group did not reach significance [trials, $F(2,1)=5.581, p=.13$; errors, $F(2,1)=$ $1.028, p=.331]$. Thus, again there is no suggestion of an effect of time of learning that might suggest a retrograde effect.

Postoperatively, the response time to stimulus presentation was examined in a $2 \times 2 \times 2$ ANOVA (set $\times$ group $\times$ sequence) with one repeated measure. Overall, there was no effect of group $[F(1,12)=0.038, p=.849]$, sequence $[F(1,12)=0.664, p=.431]$, or set $[F(1,12)=$ $0.845, p=.376]$. Equally, none of the interaction effects attained significance [group and sequence, $F(1,12)=$ $1.020, p=.333$; group and set, $F(1,12)=0.400, p=.539$; sequence and set, $F(1,12)=0.743, p=.406$; sets, sequence and group, $F(1,12)=0.779, p=.395]$. Similarly, the response times to reward delivery were compared between groups. As above, there was no effect of group $[F(1,12)=$ $0.003, p=.958]$, sequence $[F(1,12)=0.061, p=.809]$, or set $[F(1,12)=0.384, p=.547]$. Nor did the interactions between surgery and sequence $[F(1,12)=1.071$, $p=.321]$, surgery and set $[F(1,12)=0.679, p=.426]$, sequence and set $[F(1,12)=0.480, p=.502]$, and sets, sequence, and surgery $[F(1,12)=1.273, p=.281]$ attain significance. In summary, there is no indication of any differences in response times between groups, which may suggest that non-mnemonic factors were significantly influencing performance.

\section{Discussion}

The present study examined the behavioral effects of perirhinal ablation in the rat. All animals learned two sets of concurrent visual discriminations prior to bilateral perirhinal ablation or control surgery. For both groups of animals there was no significant difference between reacquisition of Set 1, learned approximately 8 weeks prior to surgery, and Set 2, learned approximately 3 weeks prior, whether tested on all data, or, more conservatively, using data from the first tested set only. However, there was evidence that the rats in the perirhinal group were impaired in reacquisition of the first set tested, regardless of when this set was learned prior to surgery. In contrast, reacquisition of the second set tested was consistently as good as that of the control animals. Together, these results suggest that perirhinal ablation in the rat leads to a reacquisition deficit limited to immediate postoperative performance. 


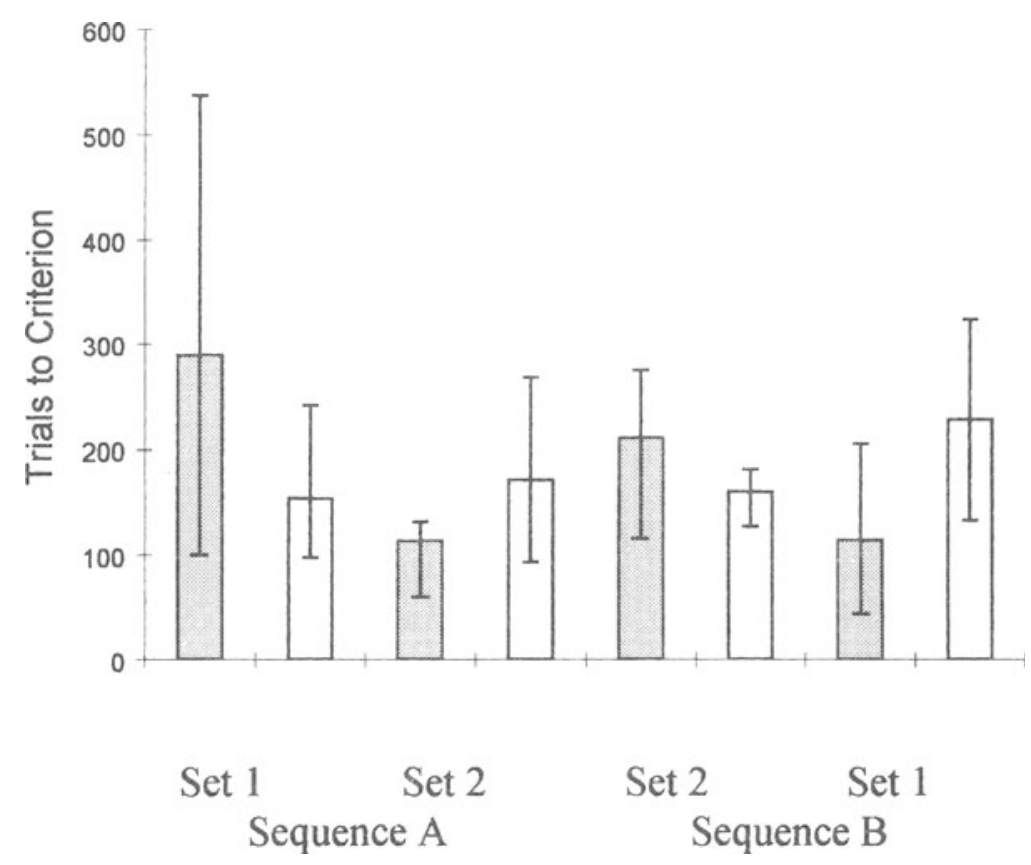

\begin{abstract}
Figure 3. Mean postoperative number of trials to criterion in retention of two sets of preoperatively learned discriminations for both the perirhinal (shaded bars) and the sham (open bars) animals. The error bars show the range of scores.
\end{abstract}

The question of whether perirhinal cortex ablation results in a temporally graded reacquisition deficit is complicated by the unexpected finding that prior to surgery Set 1 was more difficult to learn than Set 2 . Consequently, all animals in the present study had substantially more experience of the stimuli and contingencies used in Set 1 than in Set 2. It is possible that increased exposure to Set 1 aided subsequent retention and thus reacquisition. However, on the basis of previous findings, it was predicted that for the perirhinal group retention of the first-learned Set 1 would be better than that of Set 2 (Wiig et al., 1996). Had this prediction been fulfilled, it might be argued that the result may have been in part attributed to the increased exposure to Set 1 during preoperative learning. In fact, we found equivalent performance with Sets 1 and 2 in both the sham and perirhinal groups. If increased exposure to Set 1 had enhanced performance with this set, this would suggest an underlying reverse temporal gradient in the perirhinal group. Since this seems unlikely, our results suggest that, although Set 1 was more difficult to learn, this did not substantially affect its subsequent retention for either group. Indeed, a similar finding using exactly the same stimulus sets was reported in Eacott (1998), where different groups of rats learned Sets 1 and 2 , yet reacquisition rates were similar for both sets in both sham and perirhinal groups. Thus, although the difference in set difficulty is an apparently confounding factor in this analysis, there is evidence that it does not affect reacquisition rates, and thus the conclusions reached here.

However, the finding that there was a deficit limited to the first tested discrimination must also be considered here. Is it possible that this effect could have prevented a retrograde effect being observed? This suggestion would imply that any within-animal comparison of performance of Set 1 and Set 2 is flawed since testing on the second tested set followed behavior recovery of the deficit. However, a between-animals comparison of the performance on Set 1 and Set 2 when each was tested first could still reveal a greater impairment on Set 2 than Set 1 in the perirhinal group. However, there was no indication that this was the case. Thus, we conclude that there is no evidence of a temporal gradient to the retrograde effect we observed.

These findings are consistent with previous studies that failed to find a temporally graded retrograde deficit following perirhinal ablation in both the rat (Astur et al., 1995) and the monkey (Thornton et al., 1997). Contradictory reports citing a temporally graded retrograde deficit in the rat (Wiig et al., 1996) tested postoperative reacquisition of simple two-choice object discriminations learned at five different time intervals prior to surgery. It could be argued this testing procedure was not as demanding as that of the present study, which required discrimination between a larger number of stimuli presented in pseudorandomly assigned pairings, thereby making greater demands on the processes of visual identification. It is possible that this more demanding task encouraged a different learning strategy than that engaged by the rats in the study of Wiig et al. Alternatively, a greater range in the timing of preoperative learning may be necessary to reveal a temporally graded deficit. However, this seems unlikely since a similar study using more varied time in- 


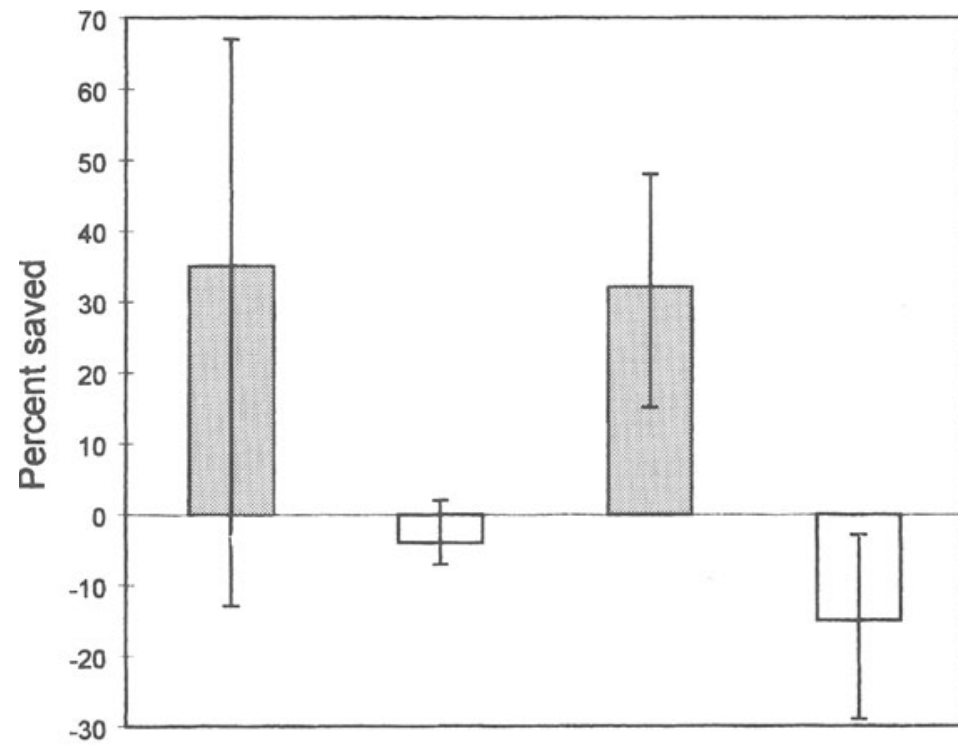

Sequence A

Sequence B

Figure 4. Mean percent savings score of postoperative trials to criterion for two sets of preoperatively learned discriminations for both the perirhinal (shaded bars) and the sham (open bars) animals. The error bars show the range of scores.

tervals also found a flat retrograde deficit in rats (Astur et al., 1995).

Although our results are consistent with previous reports of impaired retention of discrimination learning in the rat (Astur et al., 1995; Eacott, 1998; Wiig et al., 1996), they question whether the deficit is enduring in nature and whether it is due to impairments in mnemonic or nonmnemonic processes. Perirhinal ablation may disrupt a number of possible non-mnemonic processes that underlie normal performance in reacquisition of a concurrent visual discrimination task. However, no differences were found between sham and perirhinal operated animals in response times to stimuli onset, nor in those to reward delivery, even in those sets where there was a significant difference in the reacquisition rates. Therefore, although not all possible non-mnemonic deficits were tested for, our results suggest that the crucial motivational processes underlying response rates to the discriminative stimuli and reward are intact following perirhinal ablation and are not the cause of the performance decrement we observed.

The present experiment has demonstrated that perirhinal ablation in the rat produces a deficit in reacquisition limited to immediate postoperative performance. This finding is consistent with previous reports of a limited degree of behavioral recovery in animals with perirhinal lesions. Following perirhinal ablation, rats fail to discriminate between novel and familiar objects in an object discrimination task. However, the animals' performance improves substantially with increased exposure to the stimuli and length of the postoperative recovery period
(Ennaceur \& Aggleton, 1997). Therefore, behavioral recovery may be due to the animal learning to compensate for difficulties experienced in the initial postoperative testing. For example, animals may increase stimulus viewing time or focus on relearning aspects of the task. However, there is no evidence from our response time data that the animals increased their viewing time or used any other strategy that would increase response times. Although behavioral recovery has not always been seen, it is possible that previous studies did not test postoperative reacquisition sufficiently to reveal the effects of behavioral recovery.

In summary, therefore, our results suggest that it is unlikely that the perirhinal cortex contributes to a temporally graded retrieval deficit in visual discrimination learning. In addition, our results suggest that the impairment may be transitory. Thus, reports that perirhinal ablation in the rat affects acquisition and retention of visual discriminations differentially (Eacott, 1998) may be due to the order of testing postoperatively, since earlier tested discriminations are more impaired than later tested. This suggests that even new learning following perirhinal ablation may be detrimentally affected by an initial postoperative behavioral deficit in discrimination learning.

\section{EXPERIMENT 2}

The aim of Experiment 2 was to test the abilities of the operated animals to learn new concurrent visual discriminations. There are reports of both impaired new learn- 


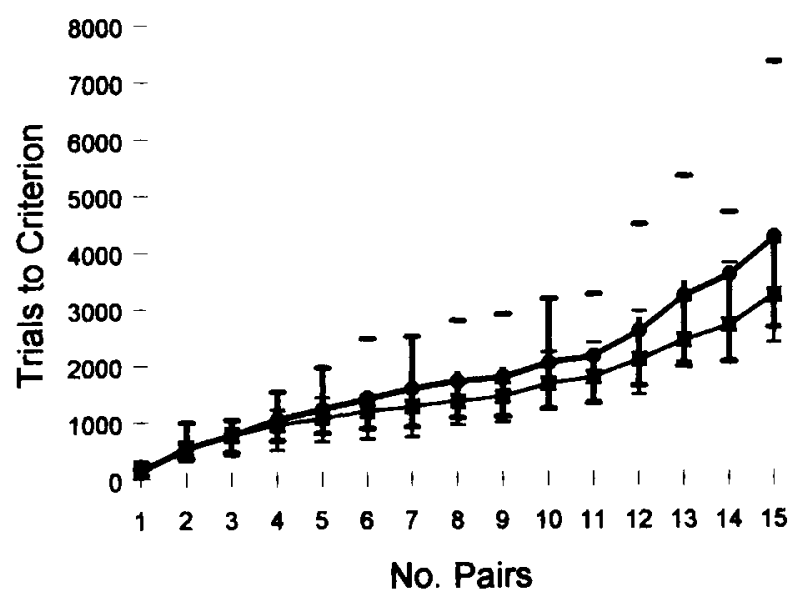

Figure 5. Number of trials to criterion in postoperative acquisition of a new set of discriminations for both the perirhinal (thin lines) and the sham (thick lines) animals. The error bars show the range of scores.

ing following perirhinal ablation in the monkey (Buckley \& Gaffan, 1997) and normal new learning in the monkey (D. Gaffan \& Murray, 1992) and the rat (Eacott, 1998). One difference between the task in which a deficit was reported (Buckley \& Gaffan, 1997) and those in which no impairment was seen (Eacott, 1998; D. Gaffan \& Murray, 1992) is the number of discriminations learned. Thus Experiment 2 was designed to place increasing demands on the processes of new discrimination learning to determine whether this would allow a deficit to be revealed. Accordingly, all animals learned a new set of concurrent visual discriminations in which the number of stimuli increased gradually up to 15 pairs.

\section{Method}

Subjects. Sixteen male Dark Agouti rats (Bantin \& Kingman, Hull, U.K.) were used in this experiment. All had undergone surgery, either sham or bilateral perirhinal ablation, and had successfully completed Experiment 1 . One rat was removed from the study after reaching criterion on five pairs of stimuli because it developed a tumor. The results of this rat are reported only up to five pairs. Therefore only 15 rats completed the study.

Apparatus. The same computer-operated $\mathrm{Y}$ maze was used as in Experiment 1.

Postoperative training. All animals learned a new set of concurrent visual discriminations, composed of 15 pairs of stimuli. As in Experiment I, in each pair of stimuli there was a rewarded stimulus, $\mathrm{S}+$, and an unrewarded foil, $\mathrm{S}-$. Acquisition of the new discrimination progressed in an almost identical manner as preoperative learning. As before, the animals were initially presented with just one pair of stimuli. When they reached the criterion of $80 \%$ correct in any given session of up to 100 trials, a new pair of stimuli was introduced, providing the subject had not accumulated more than 1,000 trials before reaching criterion in the last-learned pair. Any animal that accumulated more than 1,000 trials between pairs was removed from testing, and for the purposes of statistical analysis, 1,000 trials were added to the rat's score for the pairs it could not complete. As before, the stimulus pairings were not confined to initial pairings so that any $S+$ could be paired with any current $S-$ The rats were tested 5 days a week.

\section{Results}

The rate of acquiring the new set of discriminations postoperatively is shown in Figure 5 for both the perirhinal and sham animals. Overall, 3 sham animals and 1 perirhinal animal failed to attain criterion for all 15 pairs of stimuli (sham: R11, R9, R49; perirhinal: R50).

At five pairs, the acquisition rate for the perirhinal group was slightly better than that for the sham group, with a mean of 1,062 trials ( 270 errors) to criterion, compared with a mean of 1,214 trials ( 346 errors) to criterion for the sham group. This difference was not statistically significant [trials, $t(14)=1.02, p=.325$; errors, $t(14)=1.644, p=.122]$. At the maximum of 15 pairs, the mean number of trials to criterion for the perirhinal group was 3,218 (982 errors), compared with 4,234 trials $(1,123$ errors) for the sham group. Again, this difference did not attain statistical significance [trials, $t(13)=1.85$, $p=.087$; errors, $t(13)=1.786, p=.097]$.

\section{Discussion}

All animals in the present experiment learned a new set of concurrent visual discriminations following either bilateral perirhinal ablation or sham surgery. The animals had successfully completed the previous experiment. The number of pairs of stimuli in the concurrent discrimination task was increased from the usual 5 pairs to 15 pairs to determine whether this would have a detrimental effect on the animals' ability to acquire the discrimination task. The sham-operated animals amassed slightly more trials to attain criterion for 15 pairs of stimuli than the perirhinal group; however, this difference was not statistically significant. It appears that increasing the number of stimuli in a concurrent visual discrimination task, thereby increasing the demands on object identification, does not have a detrimental effect on acquisition following perirhinal ablation.

These results are inconsistent with previous reports indicating that increasing the number of stimuli to be learned in a concurrent visual discrimination task has a greater detrimental effect on learning in monkeys with perirhinal lesions (Buckley \& Gaffan, 1997). However, Buckley and Gaffan found impairments in postoperative acquisition by increasing the stimuli set to 80 . Similarly, they found impairments in a stimuli set of 40 when the number of foils in each pair of stimuli was increased from one to seven. It is possible that ablation of the perirhinal cortex affects acquisition of visual associative information in primates and rodents differently. However, our results are consistent with previous reports of normal acquisition of a five-pair concurrent visual discrimination task following perirhinal ablation in the rat (Eacott, 1998). An alternative explanation of our results is that we did not tax the rats' discriminative ability enough to reveal a deficit. By increasing the number of stimuli in the concurrent discrimination task further to 20 pairs or by introducing more distracting stimuli, it might have been possible to reveal a deficit. However, even with a set size of 15 pairs, the sham animals in the present experiment were beginning to fail to learn 
efficiently, with 3 animals failing to attain criterion. Therefore, it may prove unrealistic to increase the number of stimuli further. In addition, this result suggests that the rats' discriminative abilities were sufficiently taxed.

Further studies are needed to measure more discretely how visual discrimination tasks are affected by perirhinal ablation in the rat in order to determine the extent to which the perirhinal cortex is critical for the accurate identification of large numbers of visual stimuli.

\section{GENERAL DISCUSSION}

Our results have demonstrated that perirhinal ablation in the rat does not produce an enduring deficit in reacquisition of visual discrimination learning. Moreover, the ability to acquire new visual discriminations remained intact. The perirhinal animals demonstrated a significantly different pattern of reacquisition of discrimination learning than the control animals, showing a significant impairment only in reacquisition of the first discrimination tested, regardless of time since acquisition. These results suggest that the deficit we observed may be transient.

One possible interpretation of these results is that following ablation of the perirhinal cortex, learning can be accessed by an apparently highly efficient alternative system. Eacott (1998) tentatively suggested such an explanation as the reason for the impaired retention of postoperatively learned discriminations in the absence of a deficit in postoperative discrimination learning. There it was suggested that the discriminations could be learned in alternative forms and an impairment would only be apparent if a system was used for learning that was unavailable at retention test. However, this explanation is questioned by our current findings that even retention of a preoperatively acquired discrimination may be unimpaired if it is not tested as the first postoperative test. Although we do not yet have data on this question, the present results suggest that even postoperative acquisition of a new discrimination may be impaired if it is tested first. These results suggest that there is not an essential difference between retrograde and anterograde discrimination learning and the effects of perirhinal ablation. Rather, the results are more consistent with the view that the animals had an impairment, from which they recovered. The evidence from the response times of the rats to stimulus presentation and reward delivery suggests that the impairment was not a transitory disruption of the non-mnemonic processes that contribute to normal performance in the discrimination task. The mechanisms of behavioral recovery are complex and not well understood, however. Our attempts to reinstate the impairment by increasing the demands of the discrimination task were unsuccessful, in that even at a point where sham-operated animals were unable to learn efficiently, there was no effect of perirhinal ablation.

Two possible explanations of our results should be considered. First, although histology revealed that the animals all had extensive damage to perirhinal cortex, the lesions were in most cases not complete and portions of perirhinal cortex remained intact. Thus, one explanation is that an incomplete lesion had temporarily disrupted perirhinal function but did not destroy it, resulting in a transitory behavioral effect. However, although this view is difficult to disprove without testing the effects of much more extensive lesions, the remaining tissue was not extensive, and thus the idea that it could maintain normal discrimination learning to the limits of the ability of normal rats (as seen in Experiment 2) seems implausible. Note, however, that transitory effects have been reported following perirhinal cortex lesions before (Ennaceur \& Aggleton, 1997) and therefore may be a consistent feature of this type of impairment, which needs further investigation.

An alternative idea is that the task we used did not tax the central function of perirhinal cortex, although perirhinal cortex clearly contributed to performance of the task. Although our discriminative stimuli were intended to mimic junk objects, in fact they were more akin to junk scenes. Thus, the stimuli contained a number of broadly similar components that had different spatial arrangements, orientations, and patterns of contrast. In retrospect, this may have allowed the animals to solve the discrimination task other than as an object discrimination task, perhaps using spatial cues. It has been suggested that the perirhinal cortex contributes to stimulus identification by means of a perceptual learning process. In primates cells within and surrounding the perirhinal cortex have been shown to finely tune their response properties on repeat presentation of visual stimuli (primates: Sakai \& Miyashita, 1991, 1994; Tanaka, 1993; rodents: Zhu \& Brown, 1995; Zhu, Brown, \& Aggleton, 1995). Disruption to this process was thought to result in retention deficits in discrimination learning following perirhinal ablation, since the cells with response properties tuned to the task stimuli would be lost. However, if, alternatively, the task could be solved by means of the arrangement of components within a complex scene (D. Gaffan, 1994), the role of the perirhinal cortex may be less critical and therefore the effects of the lesion may be transitory.

In conclusion, therefore, our results suggest that perirhinal ablation in the rat does not produce a temporally graded retrieval deficit for visual associative information, but rather a deficit limited to initial postoperative performance. Moreover, acquisition of a new concurrent visual discrimination task is unimpaired even when the demands on the processes of identification are increased.

\section{REFERENCES}

Astur, R. S., Mumby, D. G., \& Sutherland, R. J. (1995). Perirhinal cortex damage: Effects of acquisition and retention of object and place discriminations in rats. Society for Neuroscience Abstracts, 21 , 1935.

Astur, R. S., Mumby, D. G., Weisand, M. P., \& Sutherland, R. J. (1994). Hippocampal damage in rats causes retrograde amnesia for place navigation but not object discrimination. Society for Neuroscience Abstracts, 20, 1015.

BUCKLEY, M. J., \& GAFFAN, D. (1997). Impairment of visual object dis- 
crimination learning after perirhinal ablation. Behavioral Neuroscience, 111, 467-475.

EAcoTT, M. J. (1998). Acquisition and retention of visual discrimination learning after ablation of perirhinal cortex in the rat. Psychobiology, 26, 36-41.

EacotT, M. J., Gaffan, D., \& Murray, E. A. (1994). Preserved recognition memory for small sets and impaired stimulus identification for large sets, following rhinal ablation in monkeys. European Journal of Neuroscience, 6, 1466-1478.

EAcotT, M. J., \& Heywood, C. A. (1995). Perception and memory: Action and interaction. Critical Reviews in Neurobiology, 9, 311-320.

ENNACEUR, A., \& AGgLeton, J. P. (1997). The effects of neurotoxic lesions of the perirhinal cortex combined to fornix transection on object recognition memory in the rat. Behavioural Brain Research, 80, 9-25.

Fahy, F. L., Riches, I. P., \& Brown, M. W. (1993). Neuronal activity related to visual recognition memory: Long-term memory and the encoding of recency and familiarity information in the anterior and medial inferior temporal and rhinal cortex. Experimental Brain Research, 96, 457-472.

GAFFAN, D. (1994). Dissociated effects of perirhinal ablation, fornix transection and amygdalectomy: Evidence for multiple memory systems in the primate temporal lobe. Experimental Brain Research, 99 , 411-422.

GafFan, D., \& Murray, E. A. (1992). Monkeys with rhinal cortex lesions succeed in object discrimination learning despite 24-hour intertrial intervals and fail at match to sample despite double sample presentations. Behavioral Neuroscience, 106, 30-38.

Gaffan, E. A., \& Eacott, M. J. (1995). A computer-controlled maze environment for testing visual memory in the rat. Journal of Neuroscience Methods, 60, 23-27.

Higushi, S., \& MiYashita, Y. (1996). Neural code of visual paired associate memory in primate inferotemporal cortex is impaired by perirhinal and entorhinal lesions. Proceedings of the National Academy of Sciences, 93, 739-743.

Koerner, A., Thomas, M. P., Weisand, M. P., \& Sutherland, R. J. (1996). Hippocampal-dependent memory consolidation: A comparison of three hypotheses. Society for Neuroscience Abstracts, 22 , 1118.

Kornecook, T. J., Lui, M., Duva, A. A., Anzarat, A., \& Pinel, J. P. J. (1995). Effects of perirhinal cortex lesions on object memory task in the rat. Society for Neuroscience Abstracts, 21, 1935.

Meunier, M., Bachevalier, J., Mishkin, M., \& Murray, E. A. (1993). Effects on visual recognition of combined and separate ablations of the entorhinal and perirhinal cortex in rhesus monkeys. Journal of Neuroscience, 13, 5418-5432.
Miller, E. K., Li, L., \& Desimone, R. (1993). Activity of neurons in anterior inferior temporal cortex during a short term memory task. Journal of Neuroscience, 13, 1460-1478.

Mumby, D. G., \& Pinel, J. P. J. (1994). Rhinal cortex lesions and object recognition in rats. Behavioral Neuroscience, 108, 1-8.

Paxinos, G., \& Watson, W. (1986). The rat brain in stereotaxic coordinates. London: Academic Press.

SaKaI, K., \& MiYashita, Y. (1991). Neural organisation for the longterm memory of paired associates. Nature, 354, 152-155.

SAKAI, K., \& MiYashita, Y. (1994). Visual imagery: An interaction between memory retrieval and focal attention. Trends in Neurosciences, $17,287-289$

SQuire, L. R., \& Zola-Morgan, S. (1991). The medial temporal lobe memory system. Science, 253, 1380-1386.

Tanaka, K. (1993). Neuronal mechanisms of object recognition. Science, 262, 685-688.

Thornton, J. A., Rothblat, L. A., \& Murray, E. A. (1997). Rhinal cortex removal produces amnesia for preoperatively learned discrimination problems but fails to disrupt postoperative acquisition and retention in rhesus monkeys. Journal of Neuroscience, 17, 85368549.

WARrington, E. K., \& MCCARTHY, R. A. (1988). The fractionation of retrograde amnesia. Brain \& Cognition, 7, 184-200

Weisand, M. P., Astur, R. S., \& Sutherland, R. J. (1996).The specificity and temporal characteristics of retrograde amnesia after hippocampal lesions. Society for Neuroscience Abstracts, 22, 1118.

WilG, K. A., CoOper, L., \& BEAR, M. F. (1996). Temporally graded retrograde amnesia following separate and combined lesions of the perirhinal cortex in the rat. Society for Neuroscience Abstracts, 22, 1118.

Zhu, X. O., \& Brown, M. W. (1995). Changes in neuronal activity related to the repetition and relative familiarity of visual stimuli in rhinal and adjacent cortex of the anaesthetised rat. Brain Research, 689, 101-110.

Zhu, X. O., Brown, M. W., \& Aggleton, J. P. (1995). Neuronal signalling of information important to visual recognition in rat rhinal and neighbouring cortices. European Journal of Neuroscience, 7 , 753-765.

ZOLA-Morgan, S., \& SQUiRE, L. R. (1990). The primate hippocampal formation: Evidence for a time limited role in memory storage. Science, 250, 288-289.

(Manuscript received May 28, 1998; revision accepted for publication May $24,1999$. 